\title{
AINDA O PONTO DE \\ ARTICULAÇÃO DAS SIBILANTES \\ NA ALTERAÇÃO FONOLÓGICA \\ PRIMÁRIA: DADOS DE \\ CRIANÇAS \\ PORTUGUESAS*
}

\section{LA ADQUISICIÓN DE LAS SIBILANTES EN NIÑOS PORTUGUESES CON \\ ALTERACIONES FONOLÓGICAS PRIMARIAS}

ON THE ACQUISITION OF SIBILANTS BY PORTUGUESE CHILDREN WITH

PROTRACTED PHONOLOGICAL DEVELOPMENT

Ana Margarida Ramalho**

Maria João Freitas***

Universidade de Lisboa

RESUMO: Investigação sobre o perfil fonológico das crianças portuguesas com alterações fonológicas é ainda escassa. No presente artigo, contribui-se para o avanço do trabalho nesta área, estudando-se a aquisição do contraste de ponto de articulação coronal [ \pm anterior] na classe das sibilantes, com base em dados relativos a sete crianças com alterações fonológicas primárias (associadas a perturbações dos sons da fala ou a perturbação do desenvolvimento da linguagem). Os dados foram recolhidos através da aplicação do teste CLCP-PE, tendo sido transcritos e analisados no software de análise fonológica PHON. Os resultados demonstraram a

\footnotetext{
* Esta investigação foi desenvolvida no Centro de Linguística da Universidade de Lisboa e financiada pela FCt Fundação para a Ciência e a Tecnologia, Programa UID/LIN/00214/2019

** Terapeuta da Fala, Doutorada em Linguística, Investigadora do Centro de Linguística da Universidade de Lisboa. Email: amargaridamcramalho@gmail.com

*** Linguista, Doutorada em Linguística, Professora Associada com Agregação na Universidade de Lisboa, Investigadora do Centrode Linguísticada Universidadede Lisboa. E-mail:joaofreitas@letras.ulisboa.pt.
} 
existência de padrões preferenciais e ordens de aquisição quanto à emergência e estabilização dos contrastes inerentes ao ponto de articulação das sibilantes ([-soante; -contínuo; coronal $[ \pm$ anterior $]]>>[-$ soante; +contínuo; coronal $[ \pm$ anterior $]]>>[+$ soante; +contínuo; coronal $[ \pm$ anterior $]])$ e ao vozeamento ([-vozeado] $>>$ [+vozeado]), assim como o impacto da constituência silábica (Ataque $>>$ Coda), tendo sido observada uma inesperada preferência por [s] para a Coda fricativa.

PALAVRAS-CHAVE: Português europeu. Fonologia. Traços distintivos. Sibilantes. Desenvolvimento fonológico atípico.

RESUMEN: La investigación sobre el perfil fonológico de los niños portugueses con alteraciones fonológicas es escasa por ahora. En este artículo se pretende contribuir al desarrollo de esta área estudiando la adquisición del contraste de la articulación coronal [ \pm anterior] dentro de la clase de las sibilantes. Utilizamos como punto de partida datos relativos a siete niños con alteraciones fonológicas primarias asociadas a perturbaciones en la producción de segmentos o en el desarrollo del lenguaje. Los datos han sido colectados adaptando el test CLCP al portugués europeo. Para la transcripción y análisis fonológico hemos utilizado la aplicación informática PHON. Los resultados demuestran la existencia de pautas dominantes y órdenes de adquisición en la aparición y estabilización de los contrastes inherentes al punto de articulación de las sibilantes ([-sonante; -continuo; coronal [ \pm anterior $]]>>$ [sonante; +continuo; coronal $[ \pm$ anterior $]]$ >> [+sonante; +continuo; coronal $[ \pm$ anterior $]])$ y a la sonoridad $([$ sonoro $]>>[+$ sonoro $])$, así como en el impacto de la constitución de la sílaba (Ataque >> Coda). También se ha observado una inesperada preferencia por [s] en Coda fricativa.

PALABRAS CLAVE: Portugués Europeo. Fonología. Rasgos distintivos. Sibilantes. Desarrollo fonológico atípico.

ABSTRACT: Research on the phonological profile of Portuguese children diagnosed with phonological disorders is scarce. In this paper, we intend to contribute with empirical data on the acquisition of place of articulation in sibilants (coronal [ \pm anterior]) by using the speech production of seven Portuguese children with phonological disorders (diagnosed with speech sounds disorders or with specific language impairment). The assessment test CLCP-PE was used to collect the data; the software PHON assisted the phonetic transcription and the data analysis tasks. Results showed preferential patterns, thus predicting specific orders of acquisition in the setting of the focused contrasts: (i) place of articulation: [-sonorant; - continuant; coronal [ \pm anterior]] $>>$ [-sonorant; +continuant; coronal [ \pm anterior] $]>>$ [+sonorant; +continuant; coronal [ \pm anterior $]$ ); (ii) voicing: [-voiced] >> [+voiced]); (iii) syllable constituency: Onset >> Coda (a preference for [s] for target Coda fricatives was observed).

KEYWORDS: European Portuguese. Phonological features. Sibilants. Phonological disorders.

\section{INTRODUÇÃO}

A disponibilização, na literatura especializada, de dados de produção de crianças portuguesas com desenvolvimento fonológico atípico é escassa (cf. LOUSADA 2012; RAMALHO 2017; RAMALHO, LAZAROTTO-VOLCÃO; FREITAS 2017; FREITAS; RAMALHO, 2018; REIS 2019). Com o presente estudo, pretendemos contribuir para o avanço da investigação sobre crianças com perfil fonológico atípico em português europeu (PE), descrevendo e interpretando dados recentemente recolhidos junto de crianças portuguesas diagnosticadas com alterações fonológicas primárias, decorrentes ou de perturbações dos sons da fala (PSF) (BOWEN, 2015; BROOMFIELD; DODD, 2004; CROSBIE; HOLM; DODD, 2005) ou de perturbações do desenvolvimento da linguagem (PDL) (BISHOP et al., 2016; 2017). Centrar-nos-emos na aquisição das sibilantes (/s, $\mathrm{J} / \mathrm{e} / \mathrm{z}, \mathrm{z} /$ ), dando, assim, continuidade à investigação relatada em Freitas \& Ramalho (2018), um estudo de caso sobre a perturbação fonológica em PE, no qual discutimos a aquisição do contraste de ponto de articulação [ \pm anterior] no domínio de coronal na classe das sibilantes, aspeto referido na literatura como problemático em meninos portugueses com um perfil de desenvolvimento fonológico típico (COSTA, 2010; MENDES et al., 2009/2013; AMORIM, 2014; FREITAS, 2017). A natureza problemática do contraste entre os pontos de articulação anterior e posterior é também registada em estudos sobre a perturbação fonológica na aquisição de outros sistemas linguísticos (entre outros, MATZENAUER-HERNANDORENA, 1995; DINNSEN, 1992; MOTA, 1996; LAZZAROTTO-VOLCÃO, 2009), o que justifica o seu estudo com dados do PE. 
Em PE, as sibilantes apresentam contraste entre os pontos de articulação dento-alveolar e palatal nos pares /s, J/e / $\mathrm{z}, 3 /$, bem como o contraste de vozeamento nos pares /s, z/ e /, $3 /$. O referido contraste de ponto de articulação é igualmente produtivo na classe natural das consoantes soantes, nomeadamente entre as nasais $/ \mathrm{n}, \mathrm{n} /$ e entre as laterais $(/ \mathrm{l}, \Lambda /)$. De acordo com Mateus e Andrade (2000), que adaptam a proposta de Clements e Hume (1995) ao PE, o contraste de ponto de articulação em foco é formalizado através dos traços coronal [ \pm anterior], no domínio do nó Ponto de Articulação de Consoante; o contraste de vozeamento é formalizado através de [ \pm vozeado], no domínio do nó Laríngeo. Veja-se o inventário de consoantes coronais do PE e a sua caracterização na Tabela 1:

Tabela 1: Segmentos do PE com contraste entre os pontos de articulação dento-alveolar e palatal

\begin{tabular}{c|c|c} 
& dento-alveolares & palatais \\
\hline Fricativas & $/ \mathrm{s}, \mathrm{z} /$ & $/ \int, 3 /$ \\
\hline Nasais & $/ \mathrm{n} /$ & $/ \mathrm{n} /$ \\
\hline Laterais & $/ \mathrm{l} /$ & $/ \mathrm{K} /$ \\
\hline & coronal [+anterior] & coronal $[$ - anterior]
\end{tabular}

Fonte: Adaptado de Freitas et al. (2005)

Se observarmos a distribuição silábica das sibilantes em PE, todas elas podem estar associadas a um Ataque não ramificado (cf. [s] em sim, [z] em zinco, [J] em xaile e [3] em janela); já em Coda, as variantes alofónicas de /s/, [ [, 3, z], dependem das propriedades da estrutura adjacente à direita (MATEUS; ANDRADE, 2000), tanto no domínio do nó palavra, como em contexto de sândi externo ([S] em pasta; [3] em asma; [z] em contexto de ressilabificação no Ataque vazio adjacente à direita (as amoras)).

O contraste [ \pm anterior] no domínio de coronal (ou [ \pm posterior], conforme as fontes consultadas), patente no inventário do PE registado acima, não está presente em todas as línguas do mundo, embora todos os sistemas linguísticos tenham consoantes caracterizadas como coronal [+anterior]. De acordo com Clements (2009), [coronal] encontra-se no grupo dos traços mais acessíveis e [+posterior] (ou [-anterior]) é considerado como um traço marcado pelo autor, por não estar presente em todas as línguas do mundo. Na listagem de contrastes mais comuns, Clements (2009) coloca a combinatória obstruinte posterior x anterior, que capta o contraste de ponto de articulação nos pares /s, $\mathrm{J} / \mathrm{e} / \mathrm{z}, \mathrm{3} /$, na última das quatro categorias que define na sua Escala de Robustez, aplicada à aquisição do português do Brasil em Lazzarotto-Volcão (2009) e ao PE em Amorim (2014). Tendo em conta a hierarquia de aquisição de contrastes proposta em Amorim (2014), a predição é a de que o contraste do ponto de articulação [ \pm anterior] no domínio de coronal seja problemático no percurso de aquisição do PE e mais complexo do que o contraste congénere nas soantes $/ \mathrm{n}, \mathrm{n} /$, embora o contraste no par $/ \mathrm{l}, K /$ seja o último a ser adquirido, nos dados descritos por Amorim (2014).

Vários estudos sobre o desenvolvimento fonológico típico em PE têm demonstrado uma estabilização tardia e gradual da classe natural das sibilantes em Ataque não ramificado; vejam-se os resultados, na Tabela 2, para três estudos experimentais transversais (MENDES et al., 2009,2013; AMORIM, 2014; RAMALHO, 2017). Registam-se diferenças entre os vários estudos, o que faz prever variação individual na aquisição das sibilantes em PE. No entanto, como notado em Freitas \& Ramalho (2018), as divergências poderão decorrer de questões de natureza metodológica (tipo de recolha de dados; instrumento de avaliação aplicado; taxa de sucesso usada para considerar o segmento estabilizado; tipo de transcrição dos dados), devendo os resultados ser confrontados com estudos futuros que usem metodologias espontâneas e experimentais numa mesma criança, idealmente numa perspetiva longitudinal. 
Tabela 2: Idade de aquisição das sibilantes em Ataque não ramificado em PE

\begin{tabular}{c|c|c|c|c} 
& $/ \mathrm{s} /$ & $/ \mathrm{z} /$ & $/ \mathrm{J} /$ & $/ 3 /$ \\
\hline Mendes et al. $(2009 / 2013)$ & $3 ; 0-3 ; 6$ & $4 ; 0-4 ; 6$ & $3 ; 0-3 ; 6$ & $4 ; 0-4 ; 6$ \\
\hline Amorim $(2014)$ & $3 ; 0-3 ; 5$ & $4 ; 0-4 ; 5$ & $3 ; 0-3 ; 5$ & $3 ; 0-3 ; 5$ \\
\hline Ramalho $(2017)^{1}$ & $3 ; 00-3 ; 11$ & $4 ; 00-4 ; 11$ & $4 ; 00-4 ; 11$ & $4 ; 00-4 ; 11$
\end{tabular}

Fonte: Tabela da autoria das autoras

Se observarmos os três estudos transversais experimentais (MENDES et al., 2009, 2013; AMORIM, 2014; RAMALHO, 2017), identificamos tendências para a aquisição inicial de /s, $\mathrm{J} / \mathrm{e}$ para a aquisição mais tardia das vozeadas relativamente às suas contrapartidas não vozeadas.

Dado que confrontaremos, na descrição e discussão de dados, os resultados para as sibilantes com os das coronais noutras classes naturais que exibem este tipo de segmentos (/n, n/; /l, K/; /t, d/), à imagem da metodologia adotada em Freitas \& Ramalho (2018), fornecemos, na tabela seguinte, as idades de aquisição desses segmentos nos estudos do PE supracitados:

Tabela 3: Idade de aquisição das sibilantes em Ataque não ramificado em PE

\begin{tabular}{c|c|c|c|c|c|c} 
& $/ \mathrm{n} /$ & $/ \mathrm{n} /$ & $/ \mathrm{l} / \mathrm{K} /$ & $/ \mathrm{t} /$ & $/ \mathrm{d} /$ \\
\hline $\begin{array}{c}\text { Mendes et al. (2009, } \\
\text { 2013) }\end{array}$ & $3 ; 0-3 ; 6$ & $3 ; 0-3 ; 6$ & $3 ; 6-3 ; 12$ & $3 ; 6-3 ; 12$ & $3 ; 0-3 ; 6$ & $3 ; 0-3 ; 6$ \\
\hline Amorim (2014) & $3 ; 0-3 ; 5$ & $3 ; 0-3 ; 5$ & $3 ; 0-3 ; 5$ & $4 ; 6-4 ; 11$ & $3 ; 0-3 ; 5$ & $3 ; 0-3 ; 5$ \\
\hline Ramalho (2017) & até 3;0-3;11 & $3 ; 0-3 ; 11$ & depois dos 6;0 & depois dos $6 ; 0$ & até $3 ; 0-3 ; 11$ & até $3 ; 0-3 ; 11$
\end{tabular}

Fonte: Tabela da autoria das autoras

Os dados disponíveis para a Coda /s/ referem-se apenas à variante alofónica [ $]$ ], única sibilante com este estatuto silábico contemplada nos instrumentos de avaliação aplicados em Mendes et al. (2009, 2013), Amorim (2014) e Ramalho (2017). O sumário dos resultados encontra-se na tabela 4, que disponibiliza dados apenas a partir dos três anos de idade. No entanto, com base em Freitas (1997) e em Freitas, Almeida e Costa (2012), sabemos que a aquisição das Codas fricativas pode ter início por volta dos dois anos, sendo adquirida, em primeiro lugar, a Coda fricativa em final de palavra.

\footnotetext{
${ }^{1}$ Os valores usados neste trabalho são parcialmente diferentes dos registados em Ramalho (2017), tendo sido contabilizados novamente com base no critério adotado no presente artigo relativamente à taxa de acerto para considerar um segmento adquirido (80\%), mais próxima das usadas em Mendes et al. (2009, 2013) (75\%) e em Amorim (2014) (80\%).
} 
Tabela 4: Idades de aquisição da Coda /s/ em PE

\begin{tabular}{c|c} 
& Coda $/ \mathrm{s} /-[\mathrm{S}]$ \\
\hline Mendes et al. $(2009 / 2013)$ & $3 ; 6-4 ; 0$ \\
\hline Amorim (2014) & $3 ; 0-3 ; 5$ \\
\hline Ramalho (2017) & $3 ; 0-4 ; 0$ (em aquisição) \\
& $4 ; 00-5 ; 0$ (estabilizado)
\end{tabular}

Fonte: Tabela elaborada pelas autoras

Lousada (2012), o único estudo que, tanto quanto sabemos, se debruça sobre a aquisição segmental em crianças portuguesas com diagnóstico de perturbação primária de linguagem, regista médias de percentagens de ocorrência dos processos de despalatalização e de palatalização baixas, observando-se a presença de despalatalizações em 5\% da amostra e a presença de palatalizações em 2,9\%.

No presente estudo, retomamos a questão de investigação formulada em Freitas e Ramalho (2018): de que modo uma criança portuguesa com alteração fonológica primária constrói as representações fonológicas das sibilantes da sua língua materna? Como referido então, a natureza marcada do contraste coronal [ \pm anterior] para as obstruintes nas línguas do mundo (CLEMENTS, 2001, 2009) e os dados disponíveis para o desenvolvimento típico em PE conduzem à hipótese da natureza problemática deste contraste no quadro da perturbação fonológica, embora os dados registados em Lousada (2012), sobre alterações fonológicas em crianças portuguesas com diagnóstico de perturbação primária de linguagem, levem a predizer o contrário. De modo a observar potenciais efeitos de coocorrência de traços de modo e de ponto de articulação, confrontaremos, como no estudo anterior, a aquisição do contraste em foco na aquisição dos pares $/ \mathrm{s}, \mathrm{J} / \mathrm{e} / \mathrm{z}, \mathrm{3} /$ com a aquisição do mesmo contraste nos pares /n, n/ e /l, $K /$. Por fim, descreveremos os dados em função da natureza silábica das sibilantes (Ataque ou Coda), testando potenciais efeitos deste nível prosódico na sua aquisição. A apresentação dos resultados por sujeito observado, nas secções que se seguem, justifica-se pela escassez de estudos sobre desenvolvimento fonológico em contexto atípico em PE, sendo mais prudente, na nossa opinião, não fornecer médias dos comportamentos individuais, trabalhando os dados de cada criança como estudo de caso.

\section{METODOLOGIA}

O corpus estudado na presente publicação decorre de uma amostra composta por sete sujeitos, com idades compreendidas entre os 3 anos e 2 meses e os 7 anos e 6 meses, monolingues em PE e residentes no distrito de Évora. Todos apresentam alterações fonológicas primárias, decorrentes de perturbações dos sons da fala (PSF) (BOWEN, 2015; BROOMFIELD; DODD, 2004; CROSBIE; HOLM; DODD, 2005) ou de perturbações do desenvolvimento da linguagem (PDL) (BISHOP et al., 2016, 2017), sendo todos acompanhados em terapia da fala. A criança identificada como S7 corresponde ao sujeito descrito em Freitas e Ramalho (2018). Os dados relativos a idade, género, diagnóstico e tempo de acompanhamento em terapia da fala encontram-se compilados na Tabela 5: 
Tabela 5: Características da amostra

\begin{tabular}{c|c|c|c|c} 
Código & Idade & Género & Diagnóstico & Tempo em Terapia da Fala \\
\hline S1 & $5 ; 11$ & M & PDL & $\begin{array}{r}2,5 \text { anos (com mudança de } \\
\text { terapeuta) }\end{array}$ \\
\hline S2 & $5 ; 03$ & M & PSF & 2 anos (com mudança de terapeuta) \\
\hline S3 & $3 ; 02$ & M & PSF & 2 meses \\
\hline S4 & $5 ; 05$ & M & PSF & 5 meses \\
\hline S5 & $4 ; 00$ & F & PSF & 3 anos (com mudança de terapeuta) \\
\hline S6 & $7 ; 06$ & F & PDL & 1,5 ano (com mudança de \\
terapeuta)
\end{tabular}

Fonte: Tabela elaborada pelas autoras

As crianças foram avaliadas em terapia da fala antes dos seis anos de idade, tendo sido aplicados instrumentos de avaliação adequados à faixa etária de cada, de forma a traçar o perfil de cada criança. Para tal, recorreu-se à aplicação do Teste de Avaliação da Linguagem na Criança - TALC (SUA-KAY; TAVARES, 2007), à avaliação da motricidade orofacial, sem que tenham sido identificadas alterações das estruturas e/ou funções. Nenhuma das crianças apresentava dificuldades cognitivas e/ou auditivas que justificassem as dificuldades fonológicas apresentadas.

Os procedimentos ${ }^{2}$ adotados para a recolha de dados foram semelhantes aos descritos em Freitas e Ramalho (2018), tendo sido usado o instrumento de recolha de dados CLCP-PE (RAMALHO; FREITAS; ALMEIDA, 2014), que contém o seguinte $n$ de sibilantes nas duas posições silábicas testadas (Ataque e Coda):

Tabela 6: $\mathrm{n}$ de alvos sibilantes no CLCP-PE e exemplificação

\begin{tabular}{c|c|c} 
Estruturas-alvo & $\boldsymbol{N}$ & Exemplos de estímulos \\
\hline$[\mathrm{s}]$ em Ataque & 20 & desenho de sapato \\
\hline$[\mathrm{z}]$ em Ataque & 9 & desenho de $z$ ebra \\
\hline$\left[\int\right]$ em Ataque & 10 & desenho de peixe \\
\hline$[3]$ em Ataque & 14 & desenho de girafas \\
\hline[]$]$ em Coda & 50 & desenho de castelo
\end{tabular}

Fonte: Tabela elaborada pelas autoras

\footnotetext{
${ }^{2}$ Apesar de a recolha ter sido realizada em Portugal, que não obriga a submissão ao Comitê de Ética em Pesquisa, os pais das crianças preencheram documento de consentimento informado para participação no estudo (documentos homologados pelo Comitê de Ética da University of British Columbia, Behavioural Research Ethics Board, armazenados na FLUL).
} 
Note-se que, por restrições inerentes à natureza do instrumento CLCP-PE, apenas [ $\left.\int\right]$ é testado em Coda: como nos vários testes de avaliação fonológica disponíveis para o PE, das três variantes alofónicas de /s/ em Coda, apenas [ $\left.\int\right]$ está representada porque é difícil encontrar alvos lexicais com a variante alofónica [3] em PE que integrem o léxico infantil até aos seis anos e porque a variante [z] ocorre apenas no contacto entre palavras, sendo o CLCP-PE um instrumento de nomeação de palavras ${ }^{3}$.

No que se refere à transcrição e codificação das produções das crianças, foi usado o software de análise fonológica PHON (MACWHINNEY; ROSE, 2014). Como referência de aquisição das estruturas fonológicas testadas no presente artigo, recorrer-se-á à adaptação da escala de Yavas et al. (1991): (1) taxas de acerto entre 0\% e 49\%: estrutura não adquirida; (2) taxas de acerto entre 50\% e 79\%: estrutura em aquisição; (3) taxas de acerto entre 80\% e 100\%: estrutura adquirida.

Os dados serão apresentados individualmente, mas de forma comparada, uma vez que se trata de um estudo exploratório com uma amostra limitada.

Serão fornecidas taxas de acerto da produção dos segmentos-alvo que permitem estudar o contraste de ponto de articulação (dentoalveolar versus palatal (coronal $[ \pm$ anterior $])$ ) e de vozeamento na classe das sibilantes (/s, $\int, z, 3 /$ (laríngeo $[ \pm$ vozeado])). Posteriormente, estes resultados serão comparados com os obtidos para os pares de soantes em que o mesmo contraste de ponto de articulação ocorre $(/ \mathrm{n}, \mathrm{n} / \mathrm{e} / \mathrm{l}, K /)$. Por não apresentar o contraste coronal [ \pm anterior] e por ser uma classe problemática em PE, dada a possível natureza fricativa da rótica posterior (AMORIM, 2014), a classe das vibrantes não será contemplada na nossa análise. Apresentar-se-ão, ainda, taxas de acerto para as oclusivas dento-alveolares, no sentido de observar apenas a aquisição de coronal [+anterior] em cada criança, uma vez que o contraste coronal [ \pm anterior] não existe na classe natural das oclusivas em PE.

Posteriormente, serão descritas as taxas de acerto para [ [ ] em Ataque simples e em Coda, no sentido de testar um potencial impacto da estrutura silábica na aquisição desta sibilante, a única testada neste contexto, pelas razões acima expostas.

Finalmente, e apenas no caso das sibilantes, observar-se-ão os tipos de erros encontrados e respetivas estratégias de reconstrução encontradas pelas crianças para cada estrutura.

Face à escassa dimensão da amostra, recorrer-se-á apenas à estatística não inferencial (descritiva) para a descrição dos dados, não se tendo procedido a nenhuma análise de caráter inferencial.

\section{RESULTADOS}

Nesta secção, será apresentada uma descrição dos resultados das crianças relativamente à sua produção de sibilantes durante a aplicação do CLCP-PE, a serem confrontados com os obtidos na produção de alvos segmentais em classes naturais que apresentam o contraste de ponto de articulação presente nas sibilantes. Excetuando o Gráfico 4, todos os outros gráficos apresentam valores relativos a consoantes em Ataque não ramificado.

No Gráfico 1, é possível observar as taxas de sucesso das sibilantes em Ataque não ramificado, obtidas pelas crianças testadas na amostra.

${ }^{3}$ Para mais informações sobre o instrumento e sua aplicação, consulte-se Ramalho (2017). 


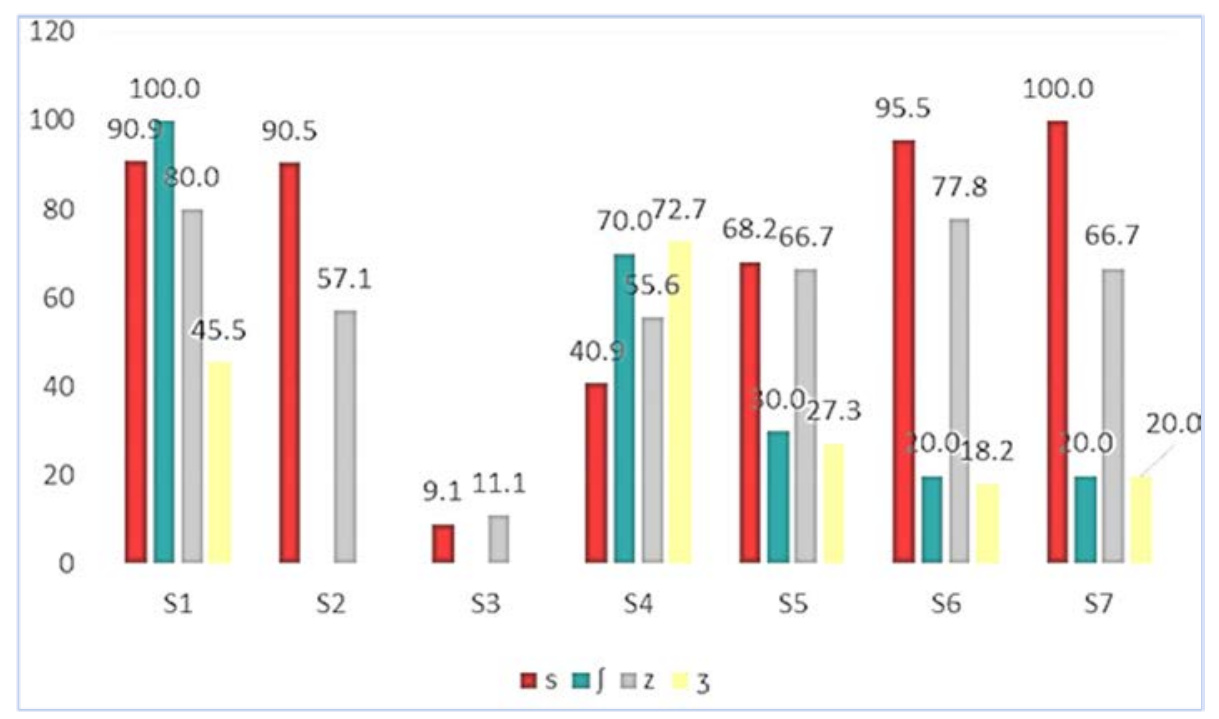

Gráfico 1: Taxas de sucesso para as sibilantes em Ataque não ramificado

Fonte: Gráfico elaborado pelas autoras

Como é possível constatar através da observação do Gráfico 1, verifica-se uma grande heterogeneidade nas produções das crianças testadas relativamente às sibilantes, embora seja possível identificar algumas tendências.

No caso de S1, verifica-se que /s/ e / / registam valores que revelam aquisição das estruturas, estando /z/ em aquisição (80\%) e não se encontrando /3/ adquirido (45,5\%). A criança S2 apenas produz fricativas dento-alveolares, estando /s/ adquirido (85,7\%) e [z] $(57,1 \%)$ em aquisição. Quanto à criança S3, esta apresenta um número residual de sibilantes (apenas /s/e /z/), não estando nenhuma delas adquiridas. O sujeito $\$ 4$ demonstra um padrão diferente, com as fricativas palatais a assumirem valores mais elevados, embora ainda em aquisição, do que as fricativas dento-alveolares, estas com taxas de acerto mais reduzidas. A criança S5 apresenta taxas de sucesso que correspondem uma aquisição em curso para /s/ e /z/, estando / $/$ e / $/ 3 /$ ainda não adquiridas, com cerca de 30\% de produções conformes ao alvo. S6 revela um comportamento semelhante, embora com um maior desfasamento entre as taxas de sucesso nas fricativas dento-alveolares ([s]: 95,5\%; [z]: 77,8\%) e nas palatais ([S]: 20\%; [3]: 18,2\%). Finalmente, na criança S7, e como já descrito em Freitas \& Ramalho (2018), apenas /s/ se encontra adquirido (100\%), sendo que /z/ se encontra em aquisição (66,7\%); quanto às palatais, estas apresentam valores correspondentes a não aquisição (20\%).

Em suma, e apesar da heterogeneidade nos comportamentos verbais das sete crianças, observa-se uma tendência generalizada para taxas de acerto superiores nas fricativas dento-alveolares, relativamente às suas congéneres palatais. De igual modo, os segmentos não vozeados tendem a corresponder a taxas de sucesso superiores ou idênticas, quando comparados com os correspondentes vozeados. Esta tendência apenas não é confirmada para a criança S4, cujo perfil demonstra o oposto.

O Gráfico 2 apresenta taxas de sucesso, por criança, relativamente às coronais nas classes das consoantes nasais e das laterais: 


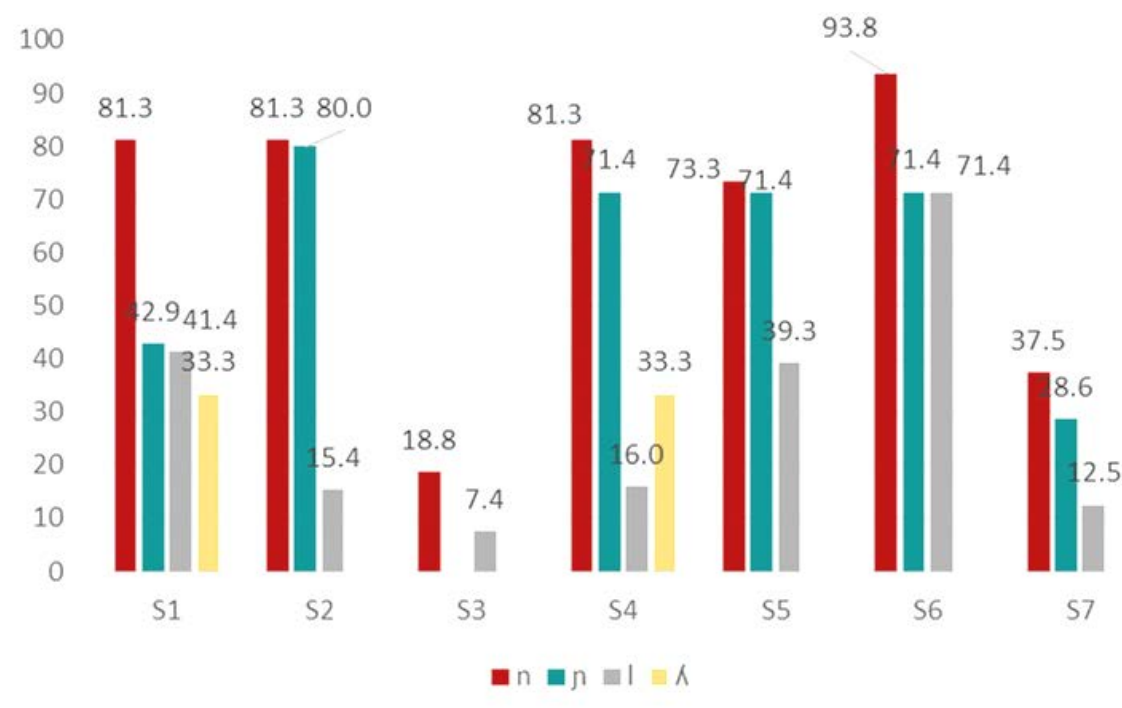

Gráfico 2: Taxas de acerto para alvos soantes (nasais e laterais) em Ataque não ramificado

Fonte: Gráfico elaborado pelas autoras

$\mathrm{Na}$ classe das soantes, apesar dos comportamentos distintos nas várias crianças, observa-se uma tendência para a emergência e estabilização de /n/ em primeiro lugar e muita dificuldade na aquisição de / $K /$, sendo que apenas duas crianças produzem instâncias fonéticas deste segmento em conformidade com o alvo, sempre com taxa de acerto baixa, de 33\%, mostrando não aquisição do segmento.

Observa-se que, para $\mathrm{S} 1$, apenas $/ \mathrm{n} /$ se encontra adquirido (81,3\%), sendo que os restantes segmentos não se encontram adquiridos ([n]: 42,9\%; [1]: 41,4\%; [K]:33,3\%). Em S2, as nasais relevam valores de estabilização, mas as líquidas não estão adquiridas, existindo apenas algumas produções para /1/ (15,4\%). Os resultados mostram que $\mathrm{S} 3$ não tem nenhuma consoante soante adquirida, sendo a única criança que não apresenta qualquer produção para /n/. Os dados relativos a $S 4$ revelam que esta criança já adquiriu /n/ e que /n/ está em aquisição, mas que as líquidas não se encontram adquiridas ([1]: 16\%; [K]):33,3\%]). Quanto a $S 5$, constata-se que as nasais se encontram em aquisição ([n]: 73,3\%; [n]: 71,4\%) e que as líquidas não se encontram adquiridas, não havendo qualquer produção para a líquida palatal. S6 revela um perfil semelhante, mas em que a nasal dento-alveolar já apresenta valores que correspondem à estabilização do segmento (93,8\%). Finalmente, S7 demonstra valores correspondentes a não aquisição de qualquer dos segmentos soantes.

Em suma, observa-se um comportamento mais estável na classe das consoantes nasais do que na das laterais. A existência de contraste entre ponto de articulação dento-alveolar e palatal regista-se para quatro das crianças na classe das nasais, o mesmo não se verificando na classe das laterais, sempre com valores abaixo dos $50 \%$.

No Gráfico 3, são apresentadas as taxas de sucesso para as oclusivas dento-alveolares, uma vez que, nesta classe do modo de articulação, $\mathrm{o} P$ E não apresenta segmentos palatais. 


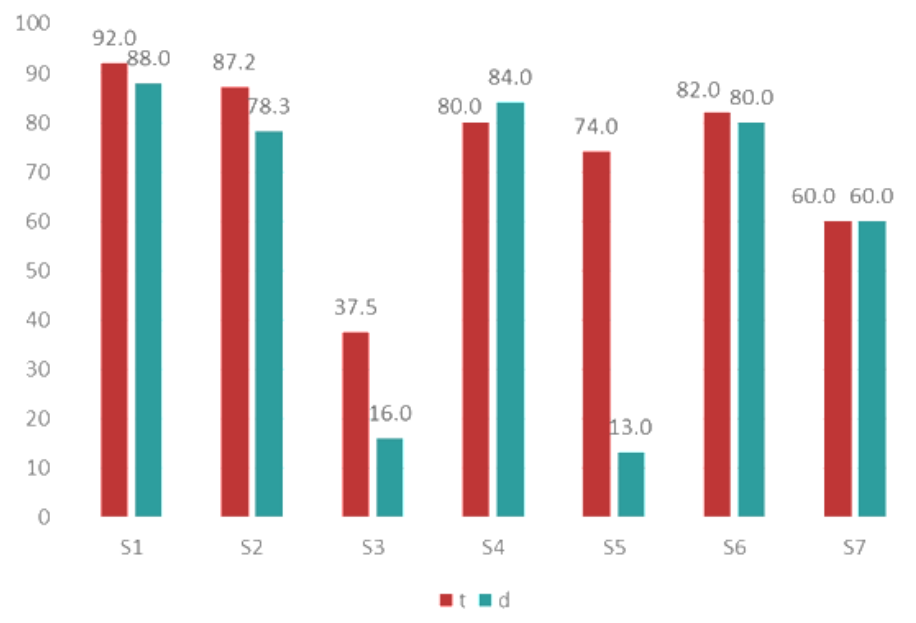

Gráfico 3: Taxas de acerto para as oclusivas dento-alveolares

Fonte: Gráfico elaborado pelas autoras

No caso das oclusivas dento-alveolares, os resultados mostram, globalmente, estabilidade no uso do ponto de articulação em foco.

Assim, S1, S4 e S6 mostram que /t/ e /d/ apresentam produções com valores correspondentes a estabilização dos segmentos, sempre acima de $80 \%$. S2 apresenta um perfil semelhante, sendo que /d/ se encontra em aquisição, mas já muito próximo da estabilização $(78,3 \%)$. No caso de $S 5$, o problema reside no contraste de vozeamento (com/t/ em aquisição, mas / d/ não adquirido) e não no ponto de articulação. Em S7, /t/ e /d/ ainda se encontram em aquisição (60\%). Uma vez mais, S3 revela problemas severos com o ponto de articulação coronal: ambas as oclusivas não se encontram adquiridas, apesar de se verificar uma produção superior para o segmento não vozeado.

No Gráfico 4, é apresentada a comparação das taxas de sucesso para a fricativa $/ \mathrm{J} /$, em Ataque não ramificado e em Coda, no sentido de testar o impacto do estatuto silábico da consoante na aquisição das sibilantes. Dadas as restrições inerentes à construção do CLCP-PE, não foi possível observar outras sibilantes em Coda (cf. secção Metodologia).

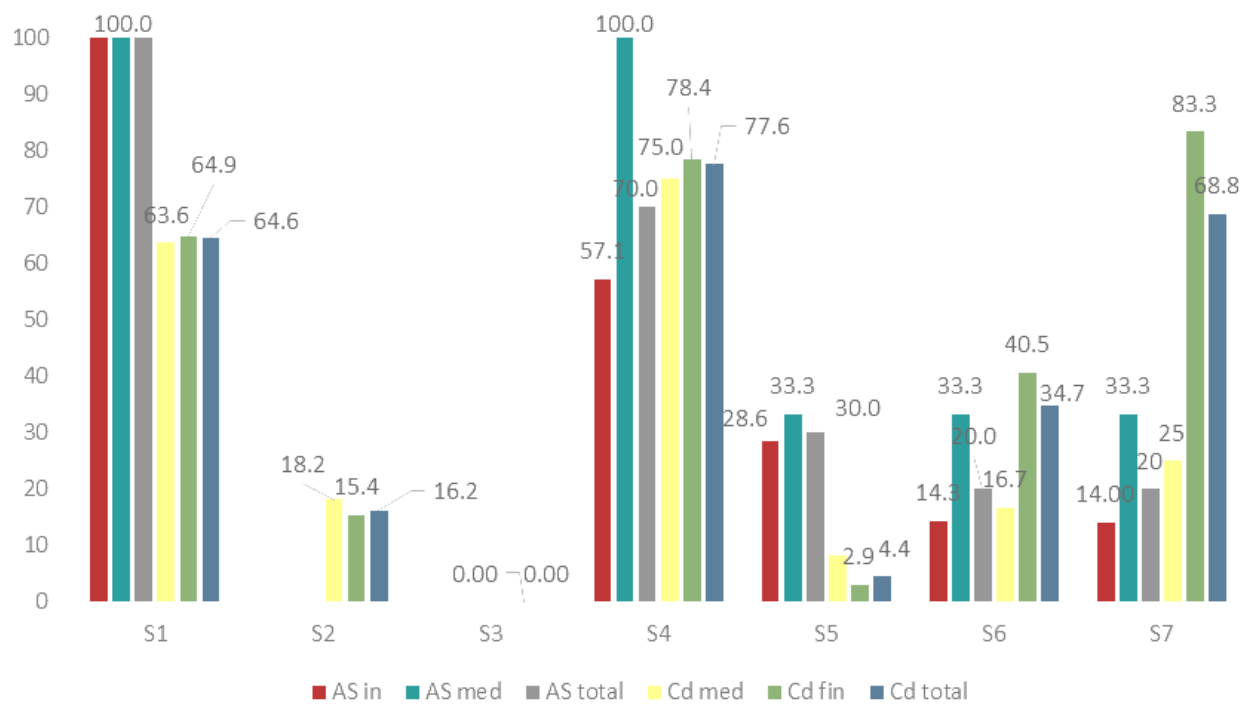

Gráfico 4: Comparação das taxas de acerto para a fricativa palatal em Ataque simples e em Coda, em função da posição na palavra 
Uma vez mais, identificamos comportamentos heterogéneos entre os sujeitos observados, embora todos, à exceção de $S 3$, que não produz [S] em conformidade com o alvo, mostrem efeitos mais (S1, S2, S5, S7) ou menos acentuados (S4, S6) do estatuto silábico da consoante.

A criança S1 apresenta estabilização da fricativa palatal em Ataque simples, apesar de esta ainda não se encontrar adquirida em Coda (com valores que rodam os 64\%, independentemente da posição na palavra). No caso de S2, a fricativa palatal não se encontra adquirida, sendo que as poucas produções de [ $\mathrm{S}]$ conformes ao alvo apenas ocorrem em Coda (medial e final). As crianças S3, S5 e S6 revelam valores que correspondem a não aquisição da estrutura em nenhuma das estruturas silábicas e posições de palavra testadas. Ainda assim, S5 privilegia o Ataque e S6 mostra uma ligeira preferência pela Coda. Em S4, globalmente, a taxa de acerto da Coda $(77,6 \%)$ é ligeiramente superior à do Ataque simples (70\%). Contudo, constata-se que o Ataque medial de palavra se encontra estabilizado (100\%), embora as restantes estruturas ainda se encontrem em aquisição. No sujeito S7, verifica-se uma tendência semelhante, em que as taxas de acerto são superiores em Coda (68,8\%), relativamente a Ataque simples (20\%). Neste caso, a Coda final regista valores correspondentes à estabilização da estrutura.

É de salientar a tendência observada para a presença de valores superiores para [ $\left.\int\right]$ em Ataque medial, quando comparado com a posição inicial de palavra, nos casos em que o segmento está presente no sistema da criança.

Seguidamente, na Tabela 7, são apresentadas as percentagens de acerto e as percentagens relativas às estratégias de reconstrução usadas pelas crianças no corpus em análise (omissões e substituições).

Tabela 7: Estratégias de reconstrução das sibilantes

\begin{tabular}{|c|c|c|c|c|c|}
\hline & & & Omissões (\%) & Substituições (\%) & Exemplos \\
\hline \multirow{5}{*}{ S1 } & $/ \mathrm{s} /$ & $90.9 \%$ & $0 \%$ & $9,1 \%$ & $\mid \mathrm{s} / \rightarrow\left[\int\right]$ \\
\hline & $\mid \mathrm{d} /$ & $100 \%$ & 0 & 0 & -- \\
\hline & $|z|$ & $80 \%$ & 0 & $20 \%$ & $|z| \rightarrow[3]$ \\
\hline & $\mid 3 /$ & $45,5 \%$ & $9,1 \%$ & $45,5 \%$ & $\begin{aligned} / 3 / & \rightarrow\left[\int\right](60 \%) \\
/ 3 / & \rightarrow[\mathrm{z}](20 \%) \\
/ 3 / & \rightarrow[\mathrm{n}](20 \%)\end{aligned}$ \\
\hline & Coda $/ \mathrm{S} /$ & $64.6 \%$ & 25 & $10.4 \%$ & $/ \mathrm{J} / \rightarrow[\mathrm{h}]$ \\
\hline \multirow{5}{*}{ S2 } & $/ \mathrm{s} /$ & $85,7 \%$ & $0 \%$ & $14,3 \%$ & $\begin{array}{c}\text { Apenas } 2 \\
/ \mathrm{J} / \rightarrow[\mathrm{v}](50 \%) \\
/ \mathrm{J} / \rightarrow[\mathrm{w}](50 \%)\end{array}$ \\
\hline & $/ \mathrm{d} /$ & $0 \%$ & $0 \%$ & $100 \%$ & $\begin{array}{l}/ \mathrm{S} / \rightarrow[\mathrm{s}](90 \%) \\
/ \mathrm{S} / \rightarrow[\mathrm{z}](10 \%)\end{array}$ \\
\hline & $|z|$ & $57,1 \%$ & $14,3 \%$ & $28,6 \%$ & $\mid \mathrm{z} / \rightarrow[\mathrm{s}]$ \\
\hline & $\mid 3 /$ & $0 \%$ & $0 \%$ & $100 \%$ & $/ 3 / \rightarrow[z]$ \\
\hline & Coda $/ \mathrm{J} /$ & $16,2 \%$ & $21,6 \%$ & $62,2 \%$ & $/ \mathrm{J} / \rightarrow[\mathrm{s}] /$ \\
\hline
\end{tabular}




\begin{tabular}{|c|c|c|c|c|c|}
\hline \multirow{5}{*}{ S3 } & $/ \mathrm{s} /$ & $9,1 \%$ & $13,6 \%$ & $77, \% 3$ & $/ s / \rightarrow[t]$ \\
\hline & $\mid \mathrm{g} /$ & $0 \%$ & $40 \%$ & $60 \%$ & $\begin{array}{c}/ \mathrm{J} / \rightarrow[\mathrm{t}](50 \%) \\
/ \mathrm{J} / \rightarrow[\mathrm{k}](16,7 \%) \\
/ \mathrm{J} / \rightarrow[\mathrm{p}](16,7 \%) \\
/ \mathrm{J} / \rightarrow[\mathrm{j}](16,7 \%)\end{array}$ \\
\hline & $|z|$ & $11,1 \%$ & $11,1 \%$ & $77,8 \%$ & $\begin{aligned}|z| & \rightarrow[t](57,1 \%) \\
|z| & \rightarrow / j](28,6 \%) \\
|z| & \rightarrow[\beta](14,3 \%)\end{aligned}$ \\
\hline & $|3|$ & $0 \%$ & $50 \%$ & $50 \%$ & $/ 3 / \rightarrow[t]$ \\
\hline & Coda / $/$ & $0 \%$ & $97,7 \%$ & $2,3 \%$ & $\mid \delta / \rightarrow[c ̧]$ \\
\hline \multirow{5}{*}{ S4 } & $/ \mathrm{s} /$ & $40,9 \%$ & $4,5 \%$ & $54,5 \%$ & $/ \mathrm{s} / \rightarrow[\mathrm{S}]$ \\
\hline & $\mid \mathrm{g} /$ & $70 \%$ & $0 \%$ & $30 \%$ & $/ S / \rightarrow[s]$ \\
\hline & $|z|$ & $55,6 \%$ & $11,1 \%$ & $33,3 \%$ & $|z| \rightarrow[3]$ \\
\hline & $|3|$ & $72,7 \%$ & $0 \%$ & $27,3 \%$ & $\begin{aligned} / 3 / & \rightarrow[\mathrm{S}](33,3 \%) \\
/ \mathrm{z} / & \rightarrow[\mathrm{r}](33,3 \%) \\
/ 3 / & \rightarrow[\mathrm{z}](33,3 \%)\end{aligned}$ \\
\hline & Coda / $/$ & $77,6 \%$ & $4,1 \%$ & $18,4 \%$ & $\mid \mathrm{S} / \rightarrow[\mathrm{s}]$ \\
\hline \multirow{5}{*}{ S5 } & $/ \mathrm{s} /$ & $68,2 \%$ & $13,6 \%$ & $18,2 \%$ & $/ \mathrm{s} / \rightarrow[\mathrm{t}] /[\mathrm{S}]$ \\
\hline & $\mid \mathrm{g} /$ & $30 \%$ & $10 \%$ & $60 \%$ & $\begin{array}{l}/ \mathrm{J} / \rightarrow[\mathrm{s}](50 \%) \\
/ \mathrm{J} / \rightarrow[\mathrm{t}](25 \%) \\
/ \mathrm{J} / \rightarrow[\mathrm{h}](25 \%)\end{array}$ \\
\hline & $|\mathrm{z}|$ & $66,7 \%$ & $11,1 \%$ & $22,2 \%$ & $\mid \mathrm{z} / \rightarrow[\mathrm{S}]$ \\
\hline & $\mid 3 /$ & $27,3 \%$ & $18,2 \%$ & $54,5 \%$ & $/ 3 / \rightarrow[S]$ \\
\hline & Coda $/ \mathrm{J} /$ & $4,4 \%$ & $88,9 \%$ & $6,7 \%$ & $\mid \mathrm{J} / \rightarrow[\mathrm{s}]$ \\
\hline \multirow{5}{*}{ S6 } & $/ \mathrm{s} /$ & $95,5 \%$ & $0 \%$ & $4,5 \%$ & $/ \mathrm{s} / \rightarrow[\mathrm{l}]$ \\
\hline & $\mid \mathrm{d} /$ & $20 \%$ & $0 \%$ & $80 \%$ & $\mid \mathrm{S} / \rightarrow[\mathrm{s}]$ \\
\hline & $|\mathrm{z}|$ & $77,8 \%$ & $0 \%$ & $22,2 \%$ & $\begin{array}{l}|z| \rightarrow[s](50 \%) \\
|z| \rightarrow[S](50 \%)\end{array}$ \\
\hline & $\mid 3 /$ & $18,2 \%$ & $0 \%$ & $81,8 \%$ & $\begin{aligned} / 3 / & \rightarrow[\mathrm{z}](55,6 \%) \\
/ 3 / & \rightarrow[\mathrm{S}](22,2 \%) \\
/ 3 / & \rightarrow[\mathrm{s}](11,1 \%) \\
/ 3 / & \rightarrow[\mathrm{d}](11,1 \%)\end{aligned}$ \\
\hline & Coda $/ \mathrm{S} /$ & $34,7 \%$ & $2 \%$ & $63,3 \%$ & $\begin{array}{c}/ \int / \rightarrow[s](96,8 \%) \\
/ S / \rightarrow[6](3,2 \%)\end{array}$ \\
\hline
\end{tabular}




\begin{tabular}{c|c|c|c|c|c}
\hline & $/ \mathrm{s} /$ & $100 \%$ & $0 \%$ & $0 \%$ & - \\
\cline { 2 - 6 } & $/ \mathrm{S} /$ & $20 \%$ & $10 \%$ & $70 \%$ & $/ \mathrm{S} / \rightarrow[\mathrm{s}]$ \\
\cline { 2 - 6 } $\mathrm{S} 7$ & $/ \mathrm{z} /$ & $77,8 \%$ & $11,1 \%$ & $11,1 \%$ & $\mid \mathrm{z} / \rightarrow[\mathrm{dz}]-$ \\
\cline { 2 - 6 } & $/ 3 /$ & $20 \%$ & $40 \%$ & $40 \%$ & $/ 3 / \rightarrow[\mathrm{z}]$ \\
\cline { 2 - 6 } & Coda $/ \mathrm{S} /$ & $68,8 \%$ & $27,1 \%$ & $4,2 \%$ & $/ \mathrm{S} / \rightarrow[\mathrm{s}]$ \\
\hline
\end{tabular}

Fonte: Tabela elaborada pelas autoras

Duas tendências se observam nas estratégias de reconstrução ativadas pelas crianças na amostra sob avaliação: (1) alternância entre sibilantes vozeadas e não vozeadas, com mais produtividade do uso de não vozeadas para alvos vozeados (sobretudo nas crianças S2, S5 e S6); (2) alternância entre os pontos de articulação dento-alveolar e palatal, sendo a tendência para produzir dento-alveolares para alvos palatais mais expressiva nas crianças S2, S5, S6 e S7. O comportamento de S3 distingue-se do das restantes crianças pelo facto de privilegiar o uso de oclusivas para alvos sibilantes, preservando tendencialmente o ponto de articulação dento-alveolar e mostrando dificuldades na aquisição das classes de modo de articulação.

Tendo em conta os constituintes silábicos em foco, é possível constatar que, na maioria dos casos, em Ataque, as taxas de omissão são inferiores às taxas de substituição. Constitui, uma vez mais, exceção a esta tendência a criança S3, que apresenta taxas de omissão elevadas para as sibilantes palatais. Já em Coda, a estratégia preferencial é a omissão (S1, S3, S5 e S7), embora os sujeitos S2, S4 e S6 usem como mais produtiva a estratégia de produção de $[\mathrm{s}]$ para o alvo $/ \mathrm{J} /$, mostrando que se encontram num estádio mais avançado de aquisição da estrutura silábica do PE.

\section{DISCUSSÃO DOS RESULTADOS}

Os resultados expostos acima revelam heterogeneidade nos comportamentos verbais das sete crianças observadas, todas diagnosticadas com alteração fonológica primária, associada a PSF ou a PDL. Com base nas propriedades fonológicas em foco, não foi possível discriminar comportamentos verbais de crianças como PDL (S1 e S6) das crianças com PSF (S2, S3, S4, S5, S7), pese embora este não fosse objetivo do presente trabalho, uma vez que os perfis das crianças em cada subgrupo (PSF e PDL) são bastante distintos em termos etários (S1 com 5;11 e S6 com 7;6) e de acompanhamento em terapia da fala (S3 com dois meses de intervenção e S6 com três anos de intervenção). Verifica-se, assim, a capacidade do CLCP-PE em discriminar S3 dos restantes sujeitos, com perfil etário e de acompanhamento em terapia da fala muito diferentes (S3 versus S1, S2, S4, S5, S6, S7).

Um aspeto crucial para o diagnóstico é o da disparidade etária entre estas crianças e os seus pares com desenvolvimento típico: notese, a título ilustrativo, que o contraste entre /s, $\mathrm{J} /$ é adquirido em faixas etárias mais baixas, nos dados de referência para o PE (na faixa etária 3;0 - 3;6 em Amorim (2014) e em Mendes et al. (2009/2013), do que aquelas em que se inserem os sujeitos da nossa amostra (entre os 4 ; e os 7;0).

Centrando-nos na relação entre representação do ponto articulação coronal e classes de modo de articulação (Gráficos 1, 2 e 3), e independentemente do contraste [ \pm anterior], as crianças observadas seguem, globalmente, o comportamento previsto com base nos dados disponíveis para o desenvolvimento fonológico típico em PE (FREITAS, 1997; AMORIM, 2014; RAMALHO, 2017): à exceção do sujeito S3 (com taxas de acerto muito baixas em todas as categorias) e do sujeito S7, todos os outros mostram a presença do traço coronal nas classes das oclusivas, das consoantes nasais e das fricativas, com taxas de acerto correspondentes à estrutura adquirida ou à estrutura em a quisição. Pelo contrário, e tal como previsto também pelos dados do desenvolvimento fonológico típico em PE, o traço coronal não está adquirido quando coocorre com [+lateral], sendo os valores de sucesso para cada categoria segmental nesta classe sempre inferiores a 50\%, em todas as crianças. Na verdade, em várias línguas do mundo, as classes das oclusivas, das 
consoantes nasais e das fricativas são as primeiras a estabilizar nos sistemas fonológicos das crianças com desenvolvimento fonológico típico (BERNHARDT; STEMBERGER, 1998, 2008; FIKKERT, 2007; FREITAS, 1997; MATZENAUERHERNANDORENA, 1990), facto que se espelha nesta observação da presença de coronais nas três classes de modo supramencionadas.

Focando-nos agora no contraste coronal [ \pm anterior], observe-se a tabela 8, que sumaria os resultados apresentados nos Gráficos 1 e 2; são registados com o símbolo $\bigotimes$ os casos de presença do contraste (em aquisição ou adquirido) e registado, em cada coluna, o segmento em aquisição ou adquirido:

Tabela 8: Sumário de dados para observação do contraste Coronal [ \pm anterior]

\begin{tabular}{|c|c|c|c|c|}
\hline & $/ \mathbf{s}, \mathbf{J} /$ & $/ \mathbf{z}, \mathbf{3} /$ & $/ \mathbf{n}, \mathbf{n} /$ & $/ \mathbf{l}, \boldsymbol{K} /$ \\
\hline S1 & $\sqrt{ }$ & $|z|$ & $/ \mathrm{n} /$ & $\varnothing$ \\
\hline S2 & $/ \mathrm{s} /$ & $|z|$ & $\sqrt{ }$ & $\varnothing$ \\
\hline S3 & $\varnothing$ & $\varnothing$ & $\varnothing$ & $\varnothing$ \\
\hline S4 & $/ \mathrm{J} /$ & $|3|$ & $\sqrt{ }$ & $\varnothing$ \\
\hline S5 & $/ \mathrm{s} /$ & $|z|$ & $\sqrt{ }$ & $\varnothing$ \\
\hline S6 & $/ \mathrm{s} /$ & $|z|$ & $\sqrt{ }$ & $/ 1 /$ \\
\hline S7 & $/ \mathrm{s} /$ & $|z|$ & $\varnothing$ & $\varnothing$ \\
\hline
\end{tabular}

Fonte: Tabela elaborada pelas autoras

Nas sibilantes (Gráfico 1; Tabela 8), e excetuando o sujeito S3, com valores de produções conformes aos alvos que não ultrapassam os 11,1\% em todas as categorias desta classe, apenas $\$ 1$ apresenta domínio do contraste em foco para /s, $\mathrm{J} /$; nos restantes sujeitos, o contraste não está adquirido e o segmento preferencial, com os perfis adquirido ou em aquisição, é o /s/ (apenas S4 tem mais sucesso $\mathrm{com} / \mathrm{f} /$ ). Comportamento semelhante é observado para o par/z, z/: o contraste coronal [ \pm anterior] não está adquirido e o segmento preferencial, com os perfis adquirido ou em aquisição, é o /z/ (apenas S4 tem mais sucesso com/z/). Os dados para as sibilantes denotam, assim, uma preferência pelo ponto de articulação coronal [+anterior], resultado nem sempre predito pelos dados do desenvolvimento típico em PE (cf. tabela 2): em Amorim (2014), a ordem de aquisição registada é / , s, z/ >>/z/; em Mendes et al. (2009/2013), a ordem é /s, J/ >>/z, 3/. Em ambos os casos, o contraste é adquirido cedo. Esta aquisição precoce do contraste coronal [ \pm anterior] nos dados do desenvolvimento fonológico típico pode ser atribuída a propriedades do input, que tornam o segmento [S] saliente (frequência no léxico; produção como marcador de plural, na periferia direita da palavra; uso de estratégias da oralidade em fronteira de palavra que privilegiam o uso de $\left[\int\right]$ e o apagamento frequente de [s] (exemplo: as sopas [ $\mathfrak{e} \int$ sópe $\left.\int\right]$ é produzido como

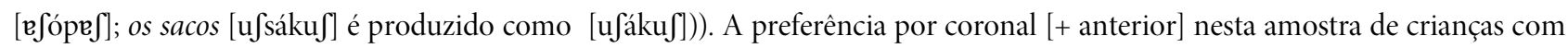
desenvolvimento atípico pode, assim, revelar menos sensibilidade aos dados do input e preferência pela ativação de uma estrutura não marcada nas línguas do mundo, universal, como é o caso da coocorrência de traços coronal [+anterior] (CLEMENTS, 2001, 2009). Note-se, porém, que Ramalho (2017) relata a ordem de aquisição / / / > /, $\mathrm{z} />>$ / 3 / no PE, a qual prevê o comportamento da amostra deste estudo (/s >> $/ \mathrm{e} / \mathrm{z}>>3 /$ ). O facto de ter sido usado o mesmo instrumento de avaliação fonológica em Ramalho (2017) e nesta investigação (o CLCP-PE), diferente dos usados em Mendes et al. (2009/2013) e em Amorim (2014), pode ser um fator relevante para justificar os dados aqui analisados; este aspeto deverá ser tido em consideração futuramente, com dados que testem dois ou mais instrumentos de avaliação fonológica na mesma amostra. 
No Gráfico 1, registam-se dois padrões quanto a [ \pm vozeado]: (1) os segmentos [-vozeado] tendem a corresponder a taxas de sucesso superiores; (2) as taxas de sucesso são idênticas para as contrapartidas [-vozeado] / [+vozeado]. Apenas a criança S4 apresenta taxas de acerto mais altas para [+vozeado]. O contraste encontra-se adquirido na maior parte dos casos, mostrando que o problema com as sibilantes reside mais na aquisição do contraste de ponto de articulação do que na aquisição do contraste de vozeamento.

Consulte-se novamente a Tabela 8 para o confronto dos dados obtidos para os pares de sibilantes /s, $\mathrm{J} / \mathrm{e} / \mathrm{z}, \mathrm{3} / \mathrm{com}$ os obtidos para os pares congéneres nas restantes classes naturais de modo observadas (/n, $\mathrm{n} / \mathrm{e} / \mathrm{l}, \Lambda /$ ). Verificamos que a aquisição do contraste coronal $[ \pm$ anterior] não ocorre da mesma forma em todas as classes observadas: (1) estabiliza primeiro na classe das consoantes nasais, o que é predito pelos dados do desenvolvimento típico do PE, com aquisição precoce desta classe (FREITAS, 1997; COSTA, 2010); (2) as sibilantes demonstram a não aquisição deste contraste, com preferência pela aquisição de inicial das dento-alveolares; (3) a classe das laterais apenas está representada em S6, com preferência pela alveolar.

Os dados acima descritos confirmam, assim, a dificuldade na aquisição do contraste obstruinte posterior x anterior predita com base em Clements (2001, 2009) e em Amorim (2014) e já evocada em Freitas e Ramalho (2018). Em Clements (2009), o contraste soante posterior $x$ anterior (neste caso, $/ \mathrm{n}, \mathrm{n} / \mathrm{e} / \mathrm{l}, K /$ ) é considerado mais acessível do que o contraste obstruinte posterior $x$ anterior (neste caso, /s, $\mathrm{J} / \mathrm{e} / \mathrm{z}, 3 /$ ), com implicações para a aquisição, também já referidas em Freitas \& Ramalho (2018): o contraste soante posterior $x$ anterior precederia, assim, a aquisição do contraste obstruinte posterior $x$ anterior. Tal foi registado na literatura para o desenvolvimento típico em PE apenas para /n, n/ (cf. Tabelas 2 e 3, acima), aspeto realçado em Amorim (2014), quando discute a proposta de Clements (2009). O contraste em $/ 1, K /$ é o último a ser adquirido em PE, dentro dos pares de segmentos em foco, facto que se confirma no presente estudo. À exceção das crianças S3 e S7, e por razões distintas (inventário fonológico muito reduzido em S3; ordem de aquisição diferente em S7), as restantes crianças da nossa amostra permitem confirmar a ordem de aquisição /n, n/ >> /s, $\mathrm{S} />>/ \mathrm{z}, 3 />>/ \mathrm{l}, \mathrm{K} /$ predita com base na literatura sobre o desenvolvimento típico em PE. Desta forma, constata-se que a coocorrência de traços [-soante; - contínuo; coronal [ \pm anterior]] precede [-soante; +contínuo; coronal [ \pm anterior]], que, por sua vez, precede [+soante; +contínuo; coronal [ \pm anterior]]. Assim, a ordem predita em Freitas \& Ramalho (2018) para as alterações fonológicas primárias, com base num estudo de caso (S7 na presente amostra) - obstruinte posterior $x$ anterior $>>$ soante posterior $x$ anterior -, não se confirma no presente estudo. A diferença poderá estar entre sujeitos com um sistema fonológico desviante (S7) e sujeitos com atraso no desenvolvimento (os restantes neste estudo).

Quanto às estratégias de reconstrução observadas no corpus em análise (alternância entre sibilantes vozeadas e não vozeadas, com mais produtividade no uso de não vozeadas para alvos vozeados, sobretudo nas crianças S2, S5 e S6; alternância entre os pontos de articulação dento-alveolar e palatal, com tendência para uso de dento-alveolares para palatais, mais expressiva nas crianças S2, S5, S6 e S7), estas revelam, tal como as taxas de acerto, a preferência pelo ponto de articulação coronal [+anterior], não marcado nas línguas do mundo, bem como pelo uso de produções [-vozeado], comportamento que obedece igualmente a uma tendência universal (CLEMENTS, 2001, 2009). No entanto, as estratégias de reconstrução permitem-nos observar dois perfis distintos: (1) crianças num estádio de desenvolvimento fonológico menos avançado, com apenas coronal [+anterior] no sistema (S2 e S7), uma vez que todas as instâncias fonéticas são dento-alveolares; (2) crianças num estádio de desenvolvimento fonológico mais avançado, com padrões de alternância que se consubstanciam em instâncias dento-alveolares para alvos palatais e vice-versa (S1, S4, S5 e S6), encontrando-se estas num estádio de instabilidade na representação fonológica dos valores do traço [ \pm anterior] no domínio de coronal. Esta avaliação é crucial para a prática clínica, pois a instabilidade associada a [ \pm anterior], com consequente não exclusividade de [+anterior], revela uma progressão no desenvolvimento e deve ser usada na programação da intervenção.

O comportamento de S3, como referimos antes, é distinto do das restantes crianças pelo facto de privilegiar o uso de oclusivas para alvos sibilantes, embora preservando tendencialmente o ponto de articulação dento-alveolar. Tal mostra que o problema nesta criança reside ainda na discriminação de modos de articulação, o que denota um conhecimento fonológico muito imaturo (FREITAS, 1997; BERNHARDT; STEMBERGER, 1998).

Quanto ao impacto da estrutura silábica na aquisição das sibilantes nesta amostra, referimos já que, em Ataque, as taxas de omissão são inferiores às taxas de substituição. A exceção, uma vez mais, é a criança S3, com taxas de omissão elevadas para as sibilantes palatais; o uso desta estratégia em Ataque tem sido associada, nos estudos sobre o PE, a um conhecimento fonológico imaturo 
(FREITAS, 1997; FREITAS; RAMALHO, 2018), perfil que se adequa a S3. Já em Coda, a estratégia preferencial é a omissão (S1, S3, S5 e S7), embora os sujeitos S2, S4 e S6 usem como mais produtiva a estratégia de produção de [s] para o alvo [S], por se encontrarem num estádio mais avançado do desenvolvimento fonológico. O uso de [s] em Coda não tem sido registado para o desenvolvimento típico em PE (FREITAS, 1997); esta preferência deverá ser testada junto de mais sujeitos com alterações fonológicas primárias, no sentido de se investigar a sua produtividade como marcador clínico na caracterização deste quadro clínico.

Os comportamentos verbais das crianças em Ataque e em Coda (para o alvo [ $\mathrm{J}$, o único que permite a comparação) mostram que os sujeitos S1, S2, S3, S5 e S7, ao contrário de S4 e S6, tratam este segmento de modo distinto nas duas posições silábicas (cf. gráfico 3 e tabela 8): (a) em Ataque, S1 mostra estabilização do segmento (100\%), o que não acontece em Coda, com 35,4\% de omissões; (b) em S2, 100\% das produções em Ataque mas apenas 62,2\% em Coda são de dento-alveolares; (c) S3 e S5 usam preferencialmente oclusivas em Ataque e omissão em Coda; (d) em S7, o segmento não está adquirido em Ataque, sendo produzido como [s], mas está em aquisição em Coda (68,8\%), sendo as formas desviantes correspondentes maioritariamente a omissão. Verifica-se, assim, nos dados sob avaliação, um impacto da estrutura silábica na aquisição em crianças com alterações fonológicas primárias. Veja-se o caso particular de S3, com um sistema fonológico muito imaturo, que usa preferencialmente oclusivas para o alvo / $/ \mathrm{em}$ Ataque, enquanto que a fricativa em Coda está sempre sujeita a omissão, o que mostra que a aquisição da estrutura silábica, sendo gradual, tem impacto na construção das representações fonológicas. Como referimos, as crianças que usam [s] em Coda estão num estádio de aquisição mais avançado do que as que apresentam omissões, uma vez que as primeiras têm já uma raiz consonântica no domínio de uma posição silábica já disponível no sistema, enquanto que as que apresentam maioritariamente omissões ainda não têm esta posição silábica disponível no seu sistema.

Referimos, acima, a tendência para a presença de valores superiores para [S] em Ataque medial, quando comparado com o Ataque inicial, nos casos em que o segmento está presente no sistema da criança. Este comportamento é inesperado, uma vez que a posição inicial de palavra é tradicionalmente assumida como um contexto facilitador da aquisição (BERNHARDT; STEMBERGER 1998, 2008). Este aspeto deverá ser testado junto de outras amostras com alteração fonológica primária, no sentido de se confirmar o efeito facilitador da posição medial de palavra na aquisição segmental em crianças com este perfil, relevante para a programação da intervenção terapêutica.

Lousada (2012) é o único estudo que descreve a aquisição segmental de crianças portuguesas com alterações fonológicas primárias. As taxas médias de ocorrência dos processos designados como despalatalização (5\%) e palatalização (2,9\%) das sibilantes na sua amostra são baixas, tendo sido registada heterogeneidade no comportamento das crianças avaliadas pela autora. Pelo contrário, $\mathrm{o}$ presente estudo mostra problemas na aquisição do contraste coronal [ \pm anterior] e taxas elevadas de alternâncias entre sibilantes dento-alveolares e palatais, o que não vai ao encontro dos resultados expostos em Lousada (2012). Dada a escassez de sujeitos portugueses com esta patologia avaliados em estudos já disponíveis, serão necessários mais dados empíricos sobre o desenvolvimento atípico em PE para verificar se a tendência registada no presente artigo é ou não recorrente nas alterações fonológicas primárias. Note-se que estudos sobre crianças brasileiras com perturbação fonológica relatam a produtividade do uso de $[\mathrm{s}$ ] e $[\mathrm{z}]$ para alvos / $/$ / e/3/, respetivamente (MATZENAUER-HERNANDORENA, 1995; MOTA, 1996; LAMPRECHT et al., 2004; LAZZAROTTO-VOLCÃO, 2009), tal como atestado na presente amostra.

\section{CONCLUSÕES}

Como referido, o presente estudo exploratório pretendeu dar continuidade ao trabalho iniciado em Freitas e Ramalho (2018), tendo como objetivo central a disponibilização de dados empíricos novos sobre a perturbação fonológica em PE, focando-nos em crianças com alterações fonológicas primárias. A heterogeneidade da amostra (idades entre os 3;2 e os 7;6), os diferentes diagnósticos específicos (PSF e PDL) e o perfil de acompanhamento em terapia da fala constituem claras limitações ao estudo. Ainda assim, foi possível formular algumas generalizações e identificar padrões preferenciais e ordens de aquisição relativas à emergência e estabilização dos contrastes inerentes às sibilantes em PE - ponto de articulação e vozeamento - bem como ao impacto da constituência silábica na aquisição do inventário fonológico do PE em contexto de desenvolvimento linguístico atípico, aspetos a testar em amostras maiores e mais homogéneas.

Ramalho \& Freitas | Ainda o ponto de articulação das sibilantes na alteração fonológica primária: dados. 


\section{REFERÊNCIAS}

AMORIM, C. Padrão de Aquisição de Contrastes do PE: a interação entre traços, segmentos e sílabas. Tese (Doutoramento em Linguística) - Faculdade de Letras, Universidade do Porto, 2014.

BERNHARDT, B. M.; STEMBERGER, J. The handbook of phonological development from the perspective of constraint-based nonlinear phonology. San Diego: Academic Press, 1998.

BERNHARDT, B. M., STEMBERGER, J. Constraint-based nonlinear phonological theories: application and implications. In: BALL, M.; PERKINGS, M.; MULLER, N.; HAWARD, S. (org.). The handbook of clinical linguistics. Oxford: Wiley-Blackwell, 2008. p. $423-437$.

BISHOP, D. V. M. et al. CATALISE: A multinational and multidisciplinary Delphi consensus study. Identifying language impairments in children. PLoS ONE, n.11, p.1-26, 2016.

BISHOP, D. V., SNOWling, M. J., THOMPSON, P. A., GREENHALGH, T., and CATALISE-2 consortium. Phase 2 of CATALISE: a multinational and multidisciplinary Delphi consensus study of problems with language development: Terminology. Journal of Child Psychology and Psychiatry and Allied Disciplines, v. 58, n. 10. p. 1068-1080. 2017.

BOWEN, C. Children's speech sound disorders. Oxford: Wiley-Blackwell, 2015.

BROOMFIELD, J.; DODD, B. The nature of referred subtypes of primary speech disability. Child Language and Therapy, v.20, n. 2, p.135-151, 2004.

CLEMENTS, G. N.; HUME, E. The internal organization of speech sounds. In: GOLDSMITH, J. (org.). The Handbook of Phonological Theory. Cambridge: Blackwell, 1995. p. 245-306.

CLEMENTS, G. N. Representational economy in constraint-based phonology. In: HALL, T. A. (ed.). Distinctive feature theory. Berlin \& New York: Mouton de Gruyter, 2001.p. 71-146.

CLEMENTS, G.N. Phonological features. In: RAIMY, E.; CAIRNS, C. E. (ed.). Contemporary views on architecture and representations in phonology. Cambridge, MA: MIT Press, 2009. p.19-68.

COSTA, T. The Acquisition of the consonantal system in European Portuguese: focus on place and manner features. 2010. Dissertação (Doutoramento) - Universidade de Lisboa, Lisboa, 2010. 
CROSBIE, S.; HOLM, A.; DODD, B. Intervention for children with severe speech disorder: a comparison of two approaches. International Journal of Language \& Communication Disorders, v.40, p.467-491, 2005.

DINNSEN, D. Variation in developing and fully developed phonetic inventories. In: FERGUSON, C.; MENN, L.; STOELGAMMON, C. (org.). Phonological development - models, research, implications. Maryland: York Press, 1992. p. 191-210.

FIKKERT, P. Acquiring phonology. In: LACY, P. de (org.). Handbook of phonological theory. Cambridge, MA: Cambridge University Press, 2007.p. 537-554.

FREITAS, M. J.; ALMEIDA, L., COSTA, T. O papel da alofonia na construção de representações lexicais em contextos monolingue e bilingue. Veredas - Revista de Estudos Linguísticos, p.83-106, 2012. Disponível em: http://www.ufjf.br/revistaveredas/edicoes/2012-2/edicao-especial-2012/. Acesso em: 22 jun. 2019.

FREITAS, M. J.; RAMALHO, M. Sobre a aquisição do ponto de articulação das sibilantes na perturbação fonológica: um estudo de caso. In: LAZZAROTTO-VOLCÃO, C.; FREITAS, M. J. (ed.). Estudos em Fonética e Fonologia - Coletânea em homenagem a Carmen Matzenauer. Curitiba: Editora CRV, 2018. p. 223-246.

FREITAS, M. J. Aquisição da estrutura silábica do português europeu. 1997. Tese (Doutoramento) - Universidade de Lisboa, Lisboa, 1997.

LAMPRECHT, R. R. et al. Aquisição fonológica do Português: perfil de desenvolvimento e subsídios para a terapia. Porto Alegre: Artmed, 2004.

LAZZAROTTO-VOLCÃO, C. Modelo padrão de aquisição de contrastes: uma proposta de avaliação e classificação dos desvios fonológicos. 2009. Tese (Doutorado) - Pontifícia Universidade Católica de Pelotas, Pelotas, 2009.

LOUSADA, M. Alterações fonológicas em crianças com perturbação de linguagem. Universidade de Aveiro. 2012. Tese (Doutoramento) - Universidade de Aveiro, Aveiro, 2012.

MACWHINNEY, B.; ROSE, Y. The PhonBank initiative. In: DURAND, J.; GUNT, U.; KRISTOFFERSEN, G. (org.). The Oxford handbook of corpus Phonology. Oxford: Oxford University Press, 2014. p. 380-401.

MATEUS, M. H.; ANDRADE, E. The phonology of oortuguese. New York: Oxford University Press, 2000.

MATZENAUER-HERNANDORENA, C. Aquisição da fonologia do português. estabelecimento de padrões com base em traços distintivos. 1990. Tese ( Doutorado) - Pontifícia Universidade Católica do Rio Grande do Sul, Porto Alegre, 1990. 
MATZENAUER-HERNANDORENA, C. Distúrbios no desenvolvimento fonológico: a relevância do traço [coronal]. Cadernos de

Estudos Linguísticos, Campinas, v. $\quad 29, \quad$ p. $\quad 69-75, \quad 1995 . \quad$ Disponível em: https://periodicos.sbu.unicamp.br/ojs/index.php/cel/article/view/8636917. Acesso em: 10 dez. 2016.

MENDES, A. et al. Teste fonético-fonológico da avaliação da linguagem pré-escolar - ALPE. Aveiro: Designeed, Lda, $2009,2013$.

MOTA, H. B. Aquisição segmental do português: um modelo implicacional de complexidade de traços. Tese (Doutorado) - Pontifícia Universidade Católica do Rio Grande do Sul, Porto Alegre, 1996.

RAMALHO, A. M. Aquisição fonológica na criança: tradução e adaptação de um instrumento de avaliação interlinguístico para o PE. 2017. Tese (Doutorado) - Universidade de Évora, Évora, 2017.

RAMALHO, A. M.; ALMEIDA, L.; FREITAS, M. J. CLCP-PE (Avaliação Fonológica da Criança: Crosslinguistic Child Phonology Project - Português Europeu). Registo IGAC: 67/2014.

RAMALHO, A. M.; LAZZAROTTO-VOLCÃO, C.; FREITAS, M. J. Contributo para a identificação de marcadores clínicos em contexto de perturbação fonológica: dados do português europeu. Matraga - Estudos Linguísticos e Literários, Rio de Janeiro, v. 24, n. 41, p. 497-523, 2017. Disponível em: https://doi.org/10.12957/matraga.2017.28714. Acesso em: 20 fev. 2018

REIS, T. A Avaliação fonológica na perturbação dos sons da fala - Modelo Padrão de Aquisição de Contrastes. Estudo de Caso. 2019. Dissertação (Mestrado) - Faculdade de Letras, Universidade de Lisboa, 2019

SUA-KAY, E.; TAVARES, D. Teste de avaliação da linguagem na criança (TALC). 4. ed. Lisboa: Oficina Didática, 2007.

YAVAS, M. et al. Avaliação fonológica da criança. Porto Alegre: Artes Médicas, 1991.

\section{(๑) $\circledast \circledast$}

Recebido em 31/01/2019. Aceito em 05/04/2019. 